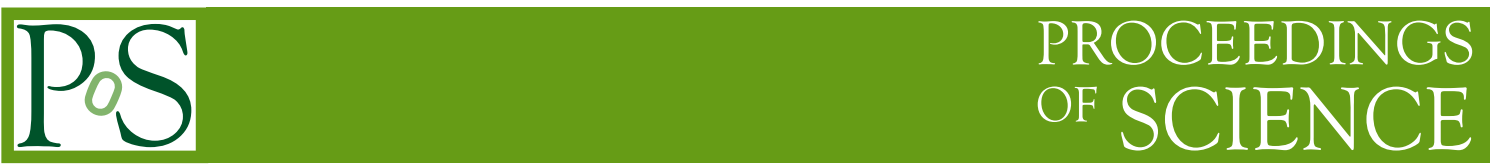

\title{
Operation and performance of the CMS tracker
}

\section{Malberti*i}

University of California Riverside

E-mail: martina.malberti@cern.ch

The CMS silicon tracker is the largest silicon detector ever built. It consists of an inner pixel detector, with 66 million readout channels, and an outer $200 \mathrm{~m}^{2}$ silicon strip detector with 10 million channels. The successful operation of this detector during the first three years of LHC operations with proton-proton and heavy ion collisions is discussed. Results include operational challenges encountered during data taking that influenced the active fraction and readout efficiency of the detectors. Details are given on the performance at high occupancy with respect to local observables, such as signal-to-noise ratio and hit reconstruction efficiency, and on radiation effects with respect to the evolution of power consumption, sensor bias, readout thresholds and leakage current.

Technology and Instrumentation in Particle Physics 2014,

2-6 June, 2014

Amsterdam, the Netherlands

* Speaker.

$\dagger$ on behalf of the CMS collaboration 


\section{Introduction}

The CMS tracker is an all-silicon detector [1] consisting of an inner pixel detector and an outer strip detector, immersed in a $3.8 \mathrm{~T}$ magnetic field, with a pseudorapidity coverage up to $|\eta|<2.5$. The sensors are arranged in concentric cylinders around the interaction region of the LHC [2] beams. Closest to the beam line is the pixel detector, which uses n-in-n silicon sensors for a total of 66 million readout channels. It is arranged in three barrel layers (BPIX) and two endcap disks per side (FPIX). The strip detector is made of 9.6 millions of silicon strips, with length between 7 and $25 \mathrm{~cm}$ and pitches of 80-205 $\mu \mathrm{m}$. It has 10 tracking layers in the barrel region and 12 endcap disks per side. The tracker provides high precision measurement of tracks with a transverse impact parameter resolution between 10 and $100 \mu \mathrm{m}$ and a momentum resolution of $1 \%$ (in the central region) to $2 \%(|\eta| \sim 2)$ for tracks in the transverse momentum range $1-10 \mathrm{GeV}$.

\section{Operation}

In the years of operation between 2010 and 2013, the LHC has delivered about $6.1 \mathrm{fb}^{-1}$ integrated luminosity of proton-proton collision data at $7 \mathrm{TeV}$ and about $23.3 \mathrm{fb}^{-1}$ at $8 \mathrm{TeV}$, reaching in 2012 a peak instantaneous luminosity of $7 \times 10^{33} \mathrm{~cm}^{-2} \mathrm{~s}^{-1}$ and up to 40 simultaneous interactions per bunch crossing [3]. Despite the increasing experimental challenges related to the increasing occupancy and radiation dose accumulated by the sensors, constant reliability was maintained over the entire running period, with $96.3 \%$ of the pixel detector and $97.5 \%$ of the strip detector channels fully operational. The main causes of failures in the pixel detector were related to poor front-end data-transfer connections and faulty analog electrical-to-optical converters. In the strip detector failures were due to short-circuits in the control rings and bias voltage lines, or due to faulty optical communications.

\section{Hit efficiency and resolution}

The first step towards high quality tracking data is the efficient and accurate reconstruction of hits from particles crossing the detector.

The hit finding efficiency, determined by measuring missing hits in well reconstructed tracks, exceeds $99 \%$ for both the strip and pixel detectors. Dynamic inefficiencies, mainly due to the saturation of the pixel Read-Out Chips (ROC) internal buffers, were observed during data-taking, with the pixel modules in the BPIX innermost layer being affected the most (Fig. 1).

The hit resolution in the pixel and strip barrel sensors has been measured by studying the distribution of the residuals between the measured and the expected hit position as predicted by the fitted tracks, with each trajectory refitted excluding the hit under study in order to minimize biases of the procedure. The intrinsic hit resolution is about $10 \mu \mathrm{m}$ in the pixel detector and varies in the strip detector between 10 and $50 \mu \mathrm{m}$, depending on the strip pitch [4]. Both pixel and strip measured hit resolutions are consistent with CMS design goals. 

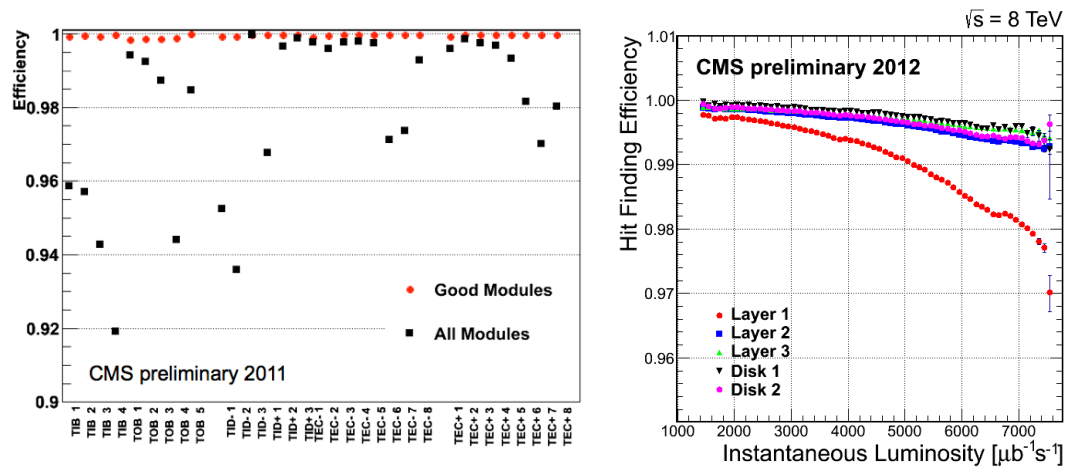

Figure 1: Hit reconstruction efficiency in different strips layers/disks (left) and pixel hit reconstruction efficiency as a function of the instantaneous luminosity (right) [4].
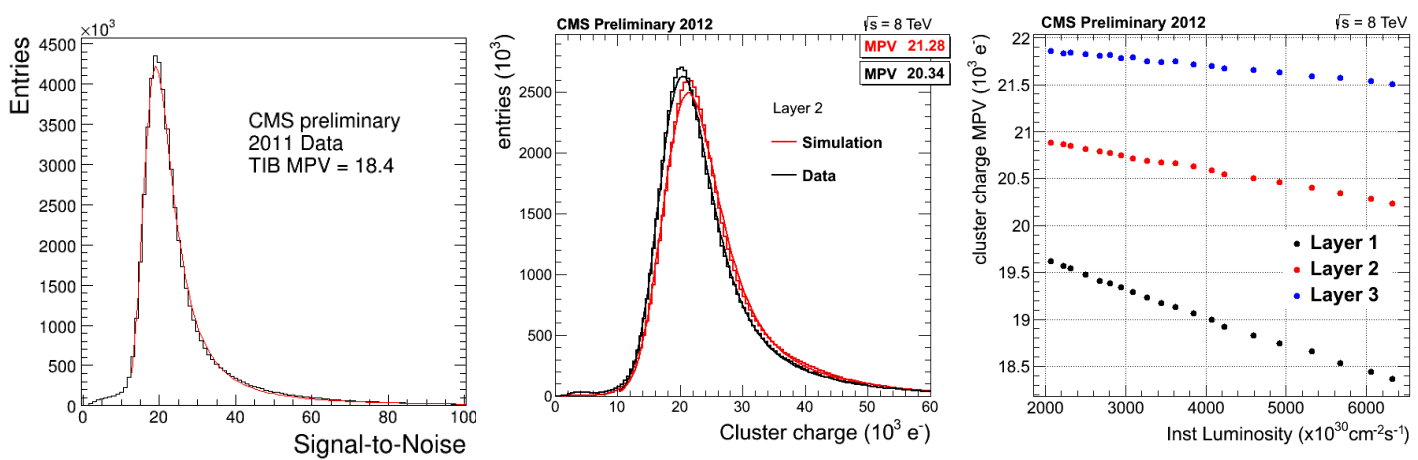

Figure 2: Signal-to-noise ratio distribution in one strip layer (left), cluster charge distribution in the pixel detector (center), and dependence of the pixel cluster charge on the instantaneous luminosity (right) [4].

\section{Charge collection}

Examples of the signal-to-noise ratio in the strip detector and cluster charge in the pixel detector are shown in Fig. 2. The distributions are fitted with the convolution of a Landau distribution and a Gaussian. As a consequence of the gain dependence on the sensor temperature and of the fact that a higher occupancy increases the power consumption and therefore the ROC temperature, a moderate dependence of the cluster charge is observed as a function of the instantaneous luminosity.

\section{Radiation induced effects}

Quantities sensitive to radiation damage, such as the leakage current, the depletion voltage and the chip operating thresholds, have been regularly monitored over the entire data-taking period. The effects are more severe for the pixel detector, especially in the inner layers, which are close to the beam pipe.

The leakage current is expected to increase as a consequence of radiation damage in the silicon bulk. A reasonable good agreement of measurements with expectations from radiation damage 

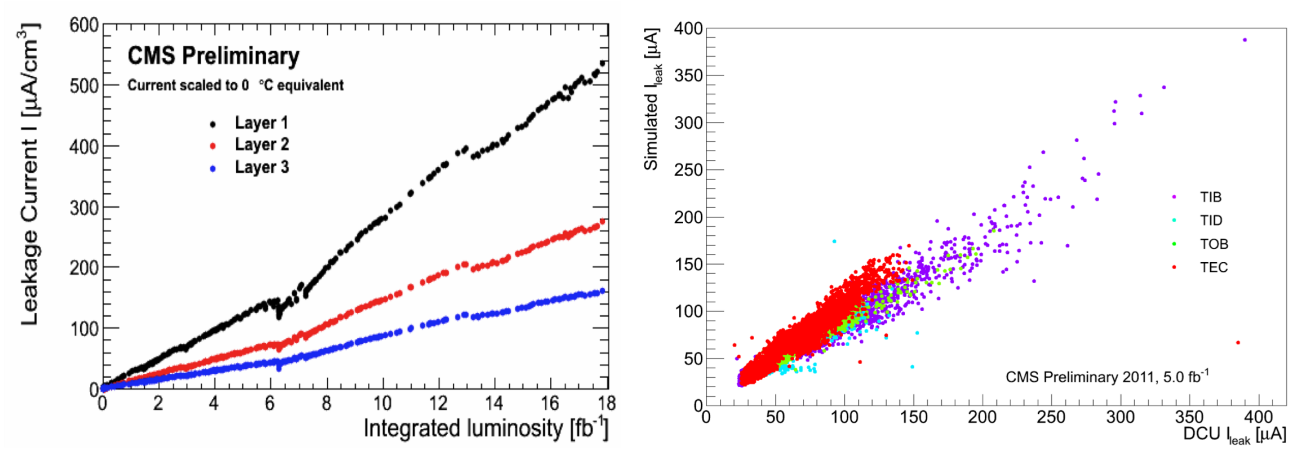

Figure 3: Leakage current as a function of the integrated luminosity in the pixel detector (left) and leakage current measured with dedicated chips (Detector Control Units or DCUs) on the front-end hybrid of the module compared to simulation in the strip detector (right) [4].
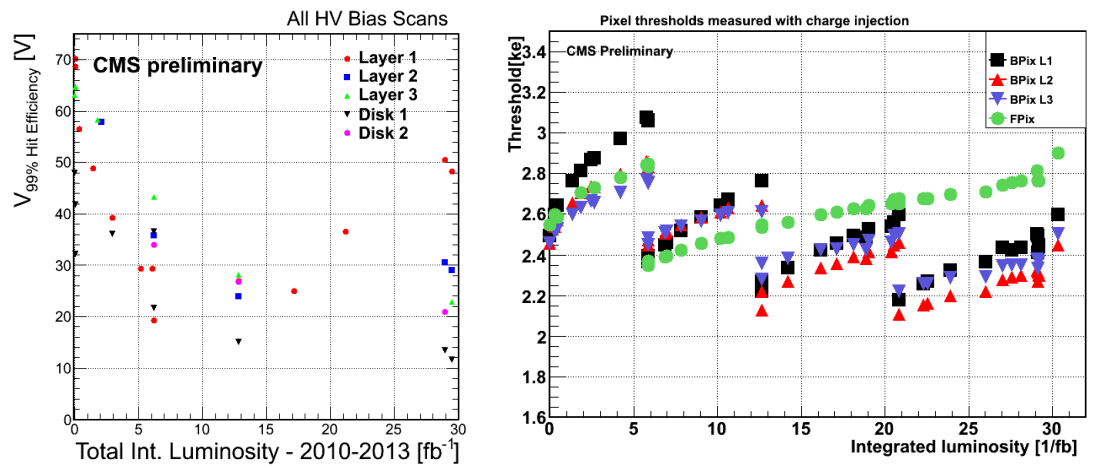

Figure 4: Voltage for which 99\% hit efficiency is obtained in the pixel detector as a function of the integrated luminosity (left) and pixel thresholds trend in 2011 and 2012 as function of the integrated luminosity (right) [4].

models was found (Fig. 3).

The bias voltage applied to the sensors during normal operation was $150 \mathrm{~V}$ and $300 \mathrm{~V}$ in the BPIX and FPIX, respectively. Dedicated scans in which the hit efficiency was measured for different values of the bias voltage were done regularly to monitor the depletion voltage. The dependence of the $99 \%$ hit efficiency on the integrated luminosity in the pixel detector is shown in Fig. 4 (left): evidence of type inversion is visible in layer 1.

Another effect observed during data-taking was the drift of the analog current and of the thresholds in the pixel detector with the integrated luminosity. A possible explanation of this effect is the bulk damage in a diode used as a reference voltage within the ROC. Since higher thresholds reduce the cluster size and charge, thresholds have been recalibrated several times during the LHC running period in order not to affect the data quality (Fig. 4 (right)).

\section{Tracking and vertexing performances}

The tracking efficiency has been measured with well identified $\mathrm{Z} \rightarrow \mu \mu$ events using a tag and 

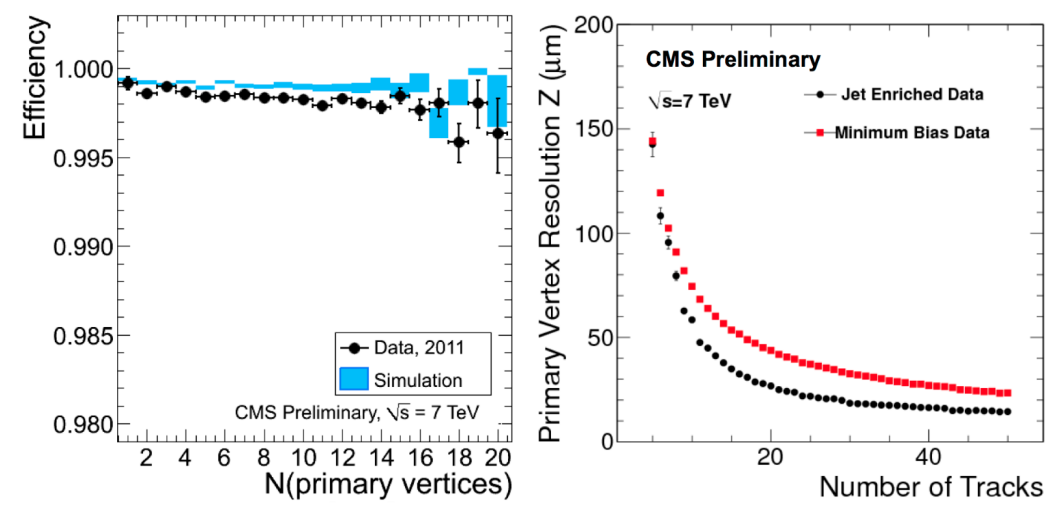

Figure 5: Tracking efficiency as a function of the number of vertices (left) and vertex resolution along the $z$ (beam) direction as a function of the number of tracks used to reconstruct the vertex (right). [5]

probe technique. The efficiency is defined as the fraction of muons reconstructed in the muon system that have a matching track in the tracker. A tracking efficiency higher than $99.5 \%$ is achieved for up to 20 interactions per event (Fig. 5 (left)).

Figure 5 (right) shows the vertex resolution along the $z$ (beam) direction as a function of the number of fitted tracks. It is measured from the difference between reconstructed vertex positions from two mutually exclusive subsets of tracks.

\section{Conclusions}

The CMS tracker detector has been successfully operated during the first LHC run, with only a small fraction of disabled channels and excellent results in the detector reliability and in tracking and vertexing performance even in high pile-up operation conditions. Improvements for the LHC Run 2 starting in 2015 are ongoing, including the repair of faulty pixel modules, which is expected to bring the fraction of faulty channels down to about $1 \%$; the lowering of the coolant temperature below $0^{\circ} \mathrm{C}$ both for pixels and strips to cope with higher luminosity operation; developments in the local reconstruction taking into account radiation induced effects.

\section{References}

[1] S. Chatrchyan et al., The CMS experiment at the CERN LHC, 2008 JINST 3 S08004

[2] L. Evans and P. Bryant, 2008 JINST 3 S08001

[3] CMS Collaboration, CMS Luminosity Public Results (2013), https://twiki.cern.ch/twiki/bin/view/CMSPublic/LumiPublicResults.

[4] CMS Collaboration, CMS Tracker Detector Performance Results (2013), https://twiki.cern.ch/twiki/bin/view/CMSPublic/DPGResultsTRK.

[5] CMS Collaboration, CMS Tracking Results (2012), https://twiki.cern.ch/twiki/bin/view/CMSPublic/PhysicsResultsTRK. 